\title{
beta-Ta and alpha-Cr thin films deposited by high power impulse magnetron sputtering and direct current magnetron sputtering in hydrogen containing plasmas
}

Hans Högberg, Lina Tengdelius, Mattias Samuelsson, Jens Jensen and Lars Hultman

\author{
Linköping University Post Print
}

\section{Tweet}

N.B.: When citing this work, cite the original article.

Original Publication:

Hans Högberg, Lina Tengdelius, Mattias Samuelsson, Jens Jensen and Lars Hultman, beta-Ta and alpha-Cr thin films deposited by high power impulse magnetron sputtering and direct current magnetron sputtering in hydrogen containing plasmas, 2014, Physica. B, Condensed matter, (439), 3-8.

http://dx.doi.org/10.1016/j.physb.2013.11.038

Copyright: Elsevier

http://www.elsevier.com/

Postprint available at: Linköping University Electronic Press

http://urn.kb.se/resolve?urn=urn:nbn:se:liu:diva-105562 


\title{
$\beta$-Ta and $\alpha$-Cr thin films deposited by high power impulse magnetron sputtering and direct current magnetron sputtering in hydrogen containing plasmas
}

Hans Högberg ${ }^{\mathrm{a}, *}$, Lina Tengdelius ${ }^{\mathrm{a}}$, Mattias Samuelsson ${ }^{\mathrm{b}}$, Jens Jensen ${ }^{\mathrm{a}}$, and Lars Hultman ${ }^{\mathrm{a}}$

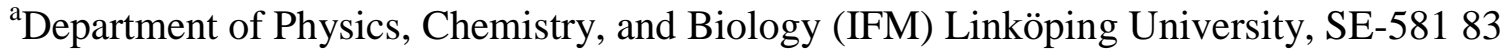
Linköping, Sweden.

${ }^{\mathrm{b}}$ Impact Coatings AB, Westmansgatan 29, SE-582 16 Linköping, Sweden

*Corresponding author. Electronic mail: hans.hogberg@liu.se

\begin{abstract}
Thin films of $\beta$-Ta and $\alpha$-Cr were deposited on $\mathrm{Si}(100)$ and $1000 \AA \mathrm{SiO}_{2} / \mathrm{Si}(100)$, by high power impulse magnetron sputtering (HiPIMS) and direct current magnetron sputtering (dcMS) in hydrogen-containing plasmas. The films were characterized by X-ray photoelectron spectroscopy (XPS), X-ray diffraction, scanning electron microscopy, elastic recoil detection analysis, and four-point probe measurements. The results showed that 001oriented $\beta$-Ta films containing up to $\sim 8$ at $\%$ hydrogen were obtained with HiPIMS, albeit with no chemical shift evident in XPS. The 110 oriented $\alpha$-Cr films display a hydrogen content less than the detection limit of 1 at $\%$, but $\mathrm{H}_{2}$ favors the growth of high-purity films for both metals. The $\beta$-Ta films deposited with dcMS are columnar, which seems independent of $\mathrm{H}_{2}$ presence in the plasma, while the films grown by HIPIMS are more fine-grained. The latter type of microstructure was present for the $\alpha-\mathrm{Cr}$ films and found to be independent on choice of technique or hydrogen in the plasma. The $\beta$-Ta films show a resistivity of $\sim 140$ $180 \mu \Omega \mathrm{cm}$, while $\alpha-\mathrm{Cr}$ films exhibit values around $30 \mu \Omega \mathrm{cm}$; the lowest values obtained for films deposited by HiPIMS and with hydrogen in the plasma for both metals.
\end{abstract}


Keywords: Hydrides, thin films, $\beta$-Ta, $\alpha$-Cr, high power impulse magnetron sputtering, direct current magnetron sputtering

\section{Introduction}

The d-block elements $\mathrm{Zr}$, Ta, and $\mathrm{Cr}$ have been reported to form hydrides as bulk materials, albeit at different temperatures and $\mathrm{H}_{2}$ pressures. For instance, when the group 4 element $\mathrm{Zr}$ in powder form is treated in 1 atmosphere of $\mathrm{H}_{2}$ at $550{ }^{\circ} \mathrm{C}$ during 2 days, the phase $\delta-\mathrm{ZrH}_{2}$ (fcc, $\mathrm{CaF}_{2}$-type structure) is formed [1]. This hydride is stable to $\sim 700-800{ }^{\circ} \mathrm{C}$ according to the phase diagram, but from literature data the transformation temperature is uncertain [2]. Moving to group 5 and the metal Ta, the hydride formation is more restricted to temperatures below or close to room temperature $[2,3]$ and with reported phases such as hexagonal $\beta-\mathrm{Ta}_{2} \mathrm{H}$ [4] and orthorhombic $\mathrm{TaH}_{0.93}$ [5]. The group 6 transition metal $\mathrm{Cr}$ is able to form hydrides with 1:1 $\mathrm{Cr}$ to $\mathrm{H}$ composition, but at a combination of high temperatures $>1000{ }^{\circ} \mathrm{C}$ and at extreme hydrogen pressure of 5.5 GPa [6]. At these conditions the metal forms two phases, one with hexagonal symmetry (hcp) and another with cubic symmetry (fcc) [6].

From the above reports, it is obvious that the transition metals $\mathrm{Zr}$, $\mathrm{Ta}$, and $\mathrm{Cr}$ interact differently with hydrogen. The trend for stability of the formed hydrides is decreasing when going from left to right in the periodic chart, which indicates a decreasing affinity to hydrogen in the order: $\mathrm{Zr}$, Ta, and $\mathrm{Cr}$. From this observation, we highlight the trend with an increasing electronegativity $(\chi)$ going from group 4 to group 6 with $\mathrm{Zr}, \chi=1.33$, Ta, 1.5 , and $\mathrm{Cr}, 1.66$ according to the Pauling scale [7]. Given the more electronegative nature of $\mathrm{H}$ with 2.2 on the Pauling scale [7] the element will act as an electron acceptor to $\mathrm{Zr}$, Ta, and $\mathrm{Cr}$ when forming a compound such as a hydride. Furthermore, the difference in electronegativity between the metal and hydrogen will determine the degree of electron transfer from the metal to hydrogen when forming a hydride, where a large difference is favorable for electron transfer. This 
suggests that hydride formation should be easiest for $\mathrm{Zr}$ and less expected for $\mathrm{Cr}$, which is in agreement with the reports above. However, the fact remains that hydrides of $\mathrm{Ta}$ and $\mathrm{Cr}$ have been synthesized. Thus, there may be alternative synthesis techniques and/or routes to grow these phases.

Thin film growth techniques such as high power impulse magnetron sputtering (HiPIMS) [8] and direct current magnetron sputtering (dcMS) are characterized by growth of materials far from thermal equilibrium. The energetic growth flux of ions in HiPIMS or neutrals in dcMS should therefore be favorable in the synthesis of $\mathrm{Ta}, \mathrm{Cr}$ as well as other hydrides such as $\delta$ $\mathrm{ZrH}_{2}[1,2]$ that are previously unstudied as thin film materials. A further advantage is the fact that films are built more or less atom-by-atom, which results in a material with well-defined properties. This is favorable to establish necessary synthesis-structure-property-relationships for these hydrides.

In this study, we investigate reactive sputtering of $\mathrm{Cr}$ and $\mathrm{Ta}$ films in hydrogen containing plasmas, using HiPIMS and dcMS and growth in an industrial scale deposition system. The deposition were performed without external heating to avoid decomposition of potential lowtemperature stable phases, using short deposition time and high power density on the target to minimize the influence of the residual gas during growth. The deposited films were investigated with respect to their chemical bonding structure, composition, structural properties, microstructure, and electrical properties.

\section{Material and methods}

The thin film growth was carried out in a commercial industrial high vacuum (HV) coating system (CemeCon $\mathrm{CC} 800 / 9 \AA)$ at a fixed target to substrate distance of $7 \mathrm{~cm}$ and with no external substrate heating. Prior to deposition the chamber was evacuated to a base pressure below $2.3 \times 10^{-4} \mathrm{~Pa}$ and to further lower the impact of the residual gas, sputtering of a titanium 
target was performed immediately before growth onto a shutter that covered the magnetron, using an effect of $1500 \mathrm{~W}$ during $600 \mathrm{~s}$ and at a pressure of $0.42 \mathrm{~Pa}$. The $\mathrm{Cr}$ films were sputtered from a chromium target with a purity of $>99.9 \%$ on $\mathrm{Si}(100)$ substrates, two in each run, while the Ta films were deposited from a tantalum target (99.5\% purity) onto $\mathrm{Si}(100)$ and $1000 \AA \mathrm{SiO}_{2} / \mathrm{Si}(100)$ substrates. Growth by HiPIMS or dcMS were conducted at a substrate bias of $-80 \mathrm{~V}$ and with the Ar partial pressure set to $0.42 \mathrm{~Pa}$ for $\mathrm{Ar} / \mathrm{H}_{2}$ plasmas containing 0, $2.5,5,10,15$, and $20 \% \mathrm{H}_{2}$ as seen from the resulting total pressure at the beginning of the deposition cycle. As no pressure control was applied for $\mathrm{H}_{2}$ during growth, the pressure increased during growth in particular for the HiPIMS processes performed with high hydrogen contents in the plasma. The power to the cathode, after ramping of the target effect behind a shutter, was set to $5000 \mathrm{~W}\left(11.36 \mathrm{~W} / \mathrm{cm}^{2}\right)$ for $\mathrm{dcMS}$ and with a $3000 \mathrm{~W}(6.82$ $\mathrm{W} / \mathrm{cm}^{2}$ ) average constant power during HiPIMS, using a pulse width of $150 \mu \mathrm{s}$ and a pulse repetition frequency of $300 \mathrm{~Hz}$. The deposition time for the $\mathrm{Cr}$ films was set to $60 \mathrm{~s}$ for the dcMS processes and $248 \mathrm{~s}$ for the HiPIMS processes, while the Ta films were grown for $138 \mathrm{~s}$ and $570 \mathrm{~s}$, for the two techniques respectively. The deposition times were selected to deposit films with similar thicknesses, using both techniques.

The chemical bonding structure present in the films was investigated by X-ray photoelectron spectroscopy (XPS), using an AXIS Ultra ${ }^{\text {DLD }}$ instrument from Kratos Analytical and analysis with monochromatic $\mathrm{Al} \mathrm{K \alpha}$ radiation. The binding energy (BE) scale was calibrated by setting the position of the Fermi edge of a sputter-cleaned $\mathrm{Ag}$ sample to $0.0 \mathrm{eV}$ [9]. The samples were sputter-cleaned by $4 \mathrm{kV} \mathrm{Ar}^{+}$at an angle of $20^{\circ}$ for $60 \mathrm{~s}$ to remove adsorbed contaminants following exposure to the air. To evaluate the amount of hydrogen incorporated in the $\mathrm{Cr}$ and Ta films as well as the level of contaminants in the $\mathrm{Cr}$ films, selected samples were investigated by elastic recoil detection analysis (ERDA). For the $\mathrm{Cr}$ samples, time-of-flight energy (ToF-E) ERDA with $36 \mathrm{MeV}{ }^{127} \mathrm{I}^{8+}$ ions as projectiles was applied to obtain the 
elemental depth profiles. The ion incident angle relative to the surface normal was $67.5^{\circ}$ and the detector was positioned at a recoil angle of $45^{\circ}$. A detailed description of the experimental set-up has been given elsewhere $[10,11]$. For the Ta samples, the hydrogen content was assessed by conventional ERDA using $3.0 \mathrm{MeV}^{4} \mathrm{He}^{+}$ions as the primary beam. An absorbing aluminium foil with a thickness of $10 \mu \mathrm{m}$ was used in front of the energy detector to filter out $\mathrm{He}^{+}$ions forward scattered due to the heavier elements. The incident angle of primary ions was $70^{\circ}$ to the sample surface normal and the recoil angle was $40^{\circ}$. A reference sample with known $\mathrm{H}$ content was used for calibration of the data in both types of ERDA measurements. The phase distributions of the films were characterized by X-ray diffraction (XRD) $\theta / 2 \theta$ scans in a Philips powder diffractometer, using $\mathrm{Cu} \mathrm{K \alpha}$ radiation at $40 \mathrm{kV}$ and $40 \mathrm{~mA}$. The film microstructures and thicknesses were investigated by cross-sectional scanning electron microscopy (SEM, LEO 1550 Gemini) operated at an acceleration voltage of $10 \mathrm{kV}$. All samples were cooled using liquid nitrogen prior to cleaving. The electrical resistivity values of the films were calculated from measured sheeth resistivity data determined from four point probe measurements Model 280C (Four dimensions) instrument and using the film thicknesses from SEM images.

\section{Results and discussion}

\subsection{Chemical bonding structure and composition}

The high-resolution XPS spectra in Fig. 1 are obtained from the $\mathrm{Cr} 2 \mathrm{p}$ and $\mathrm{Ta} 4 \mathrm{f}$ photoelectron regions: in Fig.1a from a Cr-H film deposited by HiPIMS with $20 \% \mathrm{H}_{2}$ in the plasma (solid line) and from Cr reference film grown by HiPIMS in pure Ar plasma (dashed line) and in Fig. $1 \mathrm{~b}$ from of a Ta-H film deposited by HiPIMS with $20 \% \mathrm{H}_{2}$ in the plasma (solid line) and from Ta reference film grown by HiPIMS in pure Ar plasma (dashed line). As can be seen, no chemical shifts are evident in the spectra recorded from both metals. This is in 
contrast to the chemical shift of $0.5 \mathrm{eV}$ of the $\mathrm{Zr} 3 \mathrm{~d}_{5 / 2}$ peak to higher $\mathrm{BE}$ found from analysis of synthesized $\delta-\mathrm{ZrH}_{1.9}$ compared to $\mathrm{Zr}$ powder [1]. This result suggests that both $\mathrm{Ta}$ and $\mathrm{Cr}$ interacts weakly with hydrogen, but does not rule out the possibility that hydrogen is present in the films as XPS is unable to qualitatively and quantitatively determine hydrogen. For such investigations, we applied ERDA and the measurements show that less than 1 at $\%$ hydrogen is present in $\mathrm{Cr}$ films as this amount is the detection limit for the technique. In contrast, $\sim 8$ at $\%$ of hydrogen was found in the Ta films deposited with HiPIMS and $20 \% \mathrm{H}_{2}$ in plasma, while only $\sim 4$ at $\%$ hydrogen was found in the corresponding dcMS film and with a background level of $\sim 1$ at $\%$ established from both Ta reference films. A possible explanation for this behavior is the longer deposition time applied during growth with HiPIMS, which is likely to favor diffusion of hydrogen into the film. The importance of hydrogen during growth is further supported from measurements of HiPIMS films deposited with 2.5 at $\%$ that show 4 at\% hydrogen, which is in agreement with expectations that the amount of hydrogen in the films scales with the $\mathrm{H}_{2}$ content in the plasma. The fact that higher amounts of hydrogen were found in the Ta films compared to the Cr films provides further support that Ta exhibits a higher affinity to hydrogen given its lower electronegativity value. This suggests that Ta chemically interacts with $\mathrm{H}$, but that the amount of hydrogen present in the structure is too low to yield a chemical shift in XPS and/or that the shift is very small as the chemical shift in bulk $\delta-\mathrm{ZrH}_{1.9}$ was only $\sim 0.5 \mathrm{eV}$ for the more electropositive metal $\mathrm{Zr}$ [1].

In addition, from the peak positions in Figure 1 it was possible to determine the chemical bonding in the investigated films. The measured positions with the $\operatorname{Cr} 2 \mathrm{p}$ peaks at $\mathrm{Cr} 2 \mathrm{p}_{3 / 2}=$ $574.5 \mathrm{eV}$ and $\mathrm{Cr} 2 \mathrm{p}_{1 / 2}=583.7 \mathrm{eV}$ and with the Ta $4 \mathrm{f}$ peaks at $\mathrm{Ta} 4 \mathrm{f}_{7 / 2}=21.9 \mathrm{eV}$ and $\mathrm{Ta} 4 \mathrm{f}_{5 / 2}=$ $23.8 \mathrm{eV}$ are all in excellent agreement with data established for metal-metal bonding in $\mathrm{Cr}$ and Ta [12]. In addition, the recorded high-resolution spectra from the elements $\mathrm{C}$, and $\mathrm{N}$ exhibit no visible peaks or in the case for $\mathrm{O}$ peaks of very weak intensity after $60 \mathrm{~s}$ of sputter- 
cleaning. This show that the bulk of the films is of high purity, which is further supported from the fact that lighter elements such as $\mathrm{O}$ is typically concentrated during sputter-cleaning with $\mathrm{Ar}^{+}$. The growth of high-purity Ta and $\mathrm{Cr}$ films could be confirmed by ToF-E ERDA, where the measurements of the $\mathrm{Cr}$ films showed $\mathrm{N}$ content of 0.1 at $\%$ and with slightly higher $\mathrm{O}$ and $\mathrm{C}$ contents; typically $<0.2$ at $\%$ for $\mathrm{O}$ and of $0.3-1$ at $\%$, where the highest levels encountered in films deposited with dcMS and the lowest value found for our HiPIMS film growth with $20 \% \mathrm{H}_{2}$ in plasma. The latter observation is also supported from ToF-E ERDA measurements of $\mathrm{Zr}-\mathrm{H}$ films showing a low $\mathrm{O}$ content of $<0.2$ at $\%$ in the deposited films when $\mathrm{H}_{2}$ is added to the plasma. This suggests that $\mathrm{H}_{2}$ chemically interacts with species from the residual gas present on the film surface during growth and that hydrogen decreases the level of contaminants in the deposited films. Here we note that hydrogen is frequently applied in chemical vapor deposition processes to reduce halide-based precursors [13] and to chemically etch excess carbon [14] by forming volatile species with the contaminants, thus allowing them to be removed from the growing films in the form of gaseous molecules.

\subsection{Structural properties}

The XRD patterns in Fig. 2 are recorded from Ta films deposited by HiPIMS on Si(100) substrates, with a reference film displayed in the lower diffractogram and with films grown with $10 \%$ and $20 \% \mathrm{H}_{2}$ in the plasma shown in the middle and upper diffractogram, respectively. As can be seen, all films exhibit peaks at the diffraction angles $20 \approx 17^{\circ}, 34^{\circ}, 52^{\circ}$, and $120^{\circ}$, where the peak at $34^{\circ}$ shows the highest intensity excluding the substrate 400 peak at $69^{\circ}$. These peaks originate from $\beta$-Ta, which is a metastable phase of tetragonal crystal symmetry that is frequently found for Ta films grown by HiPIMS [15] and dcMS [16]. The peaks from low to high $2 \theta$ angles can be assigned to the $001,002,003$, and 006 planes of $\beta$ Ta, where the expected 004 peak at $71^{\circ}$ is probably overlapping with the $\mathrm{Si} 400$ peak and the 005 peak that should be visible at $93^{\circ}$ is reported to be of low intensity and therefore absent 
[16]. In addition, there is a peak of low intensity located at $38^{\circ}$ that is best visible for the Ta reference film. The peak is the 110 peak from the stable bcc phase of the metal, $\alpha$-Ta. Growth of this phase is typically favored at elevated temperatures and/or on special substrates [16, 17], which is probably the main reasons why $\alpha-$ Ta films are not obtained in this study.

The fact that only peaks of the $00 \ell$ type are found in the diffractogram indicates that the films are either epitaxial or textured; the misfit to the $\mathrm{Si}(100)$ substrate is $-2.2 \%$ for 001 plane of $\beta$ Ta. However, we suggest that our films are 001 textured as films deposited on $1000 \AA$ $\mathrm{SiO}_{2} / \mathrm{Si}(100)$ substrates display $00 \ell$ peaks with comparable intensities to those recorded for films grown on $\mathrm{Si}(100)$. Furthermore, a closer inspection of the 002 peak showed a shift to lower diffraction angles when hydrogen is added to the plasma, see inset in Fig. 2. The shift was also present for films deposited on $1000 \AA \mathrm{SiO}_{2} / \mathrm{Si}(100)$ substrates. This observation suggests a larger spacing between the planes, which is most likely an effect of compressive stresses in the films given the intense ion bombardment characteristics for the HiPIMS technique [8]. We also note the difference in hydrogen content between the films with the $\sim 1$ at. $\%$, i.e. background concentration, measured for the reference film and the 8 at. $\%$ found in the film deposited with $20 \% \mathrm{H}_{2}$ in the plasma.

Furthermore, analysis of films deposited by dcMS on $\mathrm{Si}(100)$ and $1000 \AA \mathrm{SiO}_{2} / \mathrm{Si}(100)$ substrates showed that 001 oriented $\beta$-Ta films could also be grown by this technique, see Fig. 3 for films deposited on $\mathrm{Si}(100)$ and with a Ta reference film as the lower pattern and a film grown with $20 \% \mathrm{H}_{2}$ in the plasma as the upper pattern. As can been seen the 004 peak is now resolved from the Si 400 peak. The sharper peaks of lower full-width at half maximum and the change in peak positions for the $\beta$-Ta $00 \ell$ peaks to higher diffraction angles indicate a lower level of stress in the films deposited by dcMS compared to those grown by HiPIMS, but again noting the difference in hydrogen content in films grown by the two techniques. In 
addition, the fact that the 001 and 003 peaks are present in our diffraction patterns provides support to the conclusion made in [16], showing the space group of $\beta$-Ta to be $\mathrm{P}-42{ }_{1} \mathrm{~m}$ rather than the previously suggested $\mathrm{P} 4_{2} / \mathrm{mnm}[18]$.

Finally, diffraction analysis of the deposited Cr films showed that 110 oriented films of the stable bcc phase $\alpha$-Cr was obtained and with no difference in growth behavior encountered for films grown with or without $\mathrm{H}_{2}$ in the plasma or with HiPIMS or dcMS. Such texture is to be expected in sputtering of bcc metals such as Cr, see e.g. [19] and the growth condition is favored in dcMS by low substrate temperatures and at low substrate bias voltages [20] such as the conditions applied in our study.

\subsection{Microstructure}

The SEM cross-section images in Fig. 4 were obtained from $\beta$-Ta films deposited with HiPIMS for (a) a reference film and (b) a film grown with $20 \% \mathrm{H}_{2}$ in the plasma, and with dcMS for (c) a reference film and (d) a film that was processed with $20 \% \mathrm{H}_{2}$ in the plasma. From the images, a columnar growth is evident for the films deposited by dcMS whereas those grown with HIPIMS display a more fined grained microstructure, albeit with a tendency to broken columns. This growth behavior for the HiPIMS films is probably the reason for the peak broadening encountered in the diffractograms recorded compared to those seen for films deposited by dcMS as peak broadening in XRD is typically associated with a smaller grain size, see Figs. 2 and 3. The fine-grained microstructure is supported from several reports on thin film growth of metal films by HiPIMS, revealing a fine grained or even glass-like microstructure for films grown by the technique for e.g. deposition of Ta in [21] and growth of $\mathrm{Ti}$ in [22]. Furthermore, from the images it is not possible to discern any pronounced difference in microstructure between the films grown with $20 \% \mathrm{H}_{2}$ in the plasma and their corresponding reference film. This is an indication that hydrogen has little or no impact on the 
microstructural evolution of $\beta$-Ta films grown either by HiPIMS or dcMS, which finds support from the low amounts of the element that is incorporated into the films and in particular for films deposited by dcMS. In addition, the film thicknesses can be estimated from the images, to be in the range $\sim 590-630 \mathrm{~nm}$, independently of the amount of hydrogen in the plasma for the films grown with HiPIMS, whereas the dcMS films were slightly thicker with values in the range $\sim 640-690 \mathrm{~nm}$ and following the same behavior as the HiPIMS films with respect to dependence on $\mathrm{H}_{2}$ in the plasma. The corresponding growth rates could be determined to $1.1 \mathrm{~nm} / \mathrm{s}$ for the films grown by HiPIMS and more than four times higher for the films deposited by dcMS seen from a growth rate of $4.8 \mathrm{~nm} / \mathrm{s}$.

The SEM investigation showed that the $\alpha$-Cr films were considerably thinner than the $\beta$-Ta films with thicknesses in the range of $\sim 370-410 \mathrm{~nm}$ for HiPIMS films and similar values were found for the dcMS films with thicknesses in the range of $\sim 350-420 \mathrm{~nm}$, see Fig. 5. For the films deposited with dcMS there was a trend with a decreasing film thicknesses at increasing $\mathrm{H}_{2}$ content in the plasma, while no such trend was found for the films grown by HiPIMS. In spite of lower thickness values the corresponding growth rates were higher compared to $\mathrm{Ta}$ for both the HiPIMS films with $1.6 \mathrm{~nm} / \mathrm{s}$ and the dcMS films with $6.3 \mathrm{~nm} / \mathrm{s}$. The explanation is the higher sputter yield for Cr compared to Ta, with 1.1 atom/ $\mathrm{Ar}$ ion for $\mathrm{Cr}$ and 0.4 atom/ Ar ion for Ta at an ion energy of $400 \mathrm{~V}$ [23], albeit using shorter deposition time with $248 \mathrm{~s}$ for HiPIMS and $60 \mathrm{~s}$ for dcMS The images show no pronounced difference in microstructure between the films deposited by HiPIMS and dcMS or with or without $\mathrm{H}_{2}$ in the plasma as all investigated films show similar fine grained microstructure with tendency to form columns. The reason for this behavior could be the higher sputter yield of $\mathrm{Cr}$ compared to Ta, which leads to fast nucleation at the applied growth conditions that are characterized by low surface mobility as no external heating was applied. 


\subsection{Resistivity}

Four-point probe measurements showed that the deposited $\alpha$-Cr and $\beta$-Ta films were metallic conductors. All $\alpha$-Cr films display resistivity values $\sim 30 \mu \Omega \mathrm{cm}$ irrespective of deposition technique and if hydrogen has been added to the plasma or not, but with the lowest values determined for films deposited by HiPIMS and with hydrogen in the plasma. Here, we note that these films exhibited a low level of contaminants according to the ToF-E ERDA measurements, supporting properties of hydrogen in decreasing the incorporation of contaminants during film growth. The resistivity values obtained are about double that of bulk $\alpha-\mathrm{Cr}$ with a resistivity of $12.9 \mu \Omega \mathrm{cm}$ [24]. This can be expected as thin films generally display a higher resistivity values due to a more pronounced surface scattering.

The $\beta$-Ta films showed resistivity values in the range of $\sim 140-180 \mu \Omega \mathrm{cm}$, which is in agreement with previous studies reporting values of $180-220 \mu \Omega \mathrm{cm}$, see review in [17]. These values are about ten times higher than for of bulk $\alpha$-Ta with a resistivity of $13.1 \mu \Omega \mathrm{cm}$ [24] and reflect the electrical properties of $\beta$-Ta films. Consequently, the high resistivity value of $\beta$-Ta films constitutes a limitation in applications envisioned for Ta films as, for instance, high temperature stable conductive films. As for the metal $\mathrm{Cr}$, the lowest values were measured for films deposited by HiPIMS and using hydrogen in the plasma. In contrast, the films grown by dcMS showed slightly higher resistivity values when hydrogen was added to the plasma. This suggests that the shorter deposition time of $138 \mathrm{~s}$ applied for growth by dcMS compared to $570 \mathrm{~s}$ applied in HiPIMS provides hydrogen with shorter time to chemically remove contaminants from the growing film. 


\section{Conclusions}

Our study shows that sputtering of $\mathrm{Ta}$ and $\mathrm{Cr}$ in a $\mathrm{H}_{2}$-containing plasma by the HiPIMS and dcMS techniques results in the growth of 001 oriented $\beta$-Ta films on bare and $\mathrm{SiO}_{2}$-covered $\operatorname{Si}(100)$ wafer substrates, and 110 -oriented $\alpha$-Cr films deposited on $\operatorname{Si}(100)$. The $\beta$-Ta films contain up to 8 at $\%$ of hydrogen when grown with HiPIMS using $20 \% \mathrm{H}_{2}$ in the plasma. While the amount of hydrogen in the as-deposited $\mathrm{Cr}$ films is below the detection limit, the hydrogen in the discharge favors growth of films with a very low level $\mathrm{C}, \mathrm{N}$, and $\mathrm{O}$ with less than 0.2 at $\%$ of oxygen determined for the $\mathrm{Cr}$ films. The $\beta$-Ta films deposited by dcMS exhibit a columnar microstructure independent of $\mathrm{H}_{2}$ in the plasma, while the films grown with HiPIMS display a more fine-grained microstructure characteristic to this technique. The resistivity of the $\beta$-Ta films is in the range of $\sim 140-180 \mu \Omega \mathrm{cm}$ and agrees with previously reported values, while the $\mathrm{Cr}$ films exhibit a lower resistivity of $\sim 30 \mu \Omega \mathrm{cm}$, i.e. twice that of the bulk metal.

\section{Acknowledgments}

Dr. Lars-Åke Näslund is gratefully acknowledged for assistance with the XPS measurements. $\mathrm{HH}$ acknowledges financial support from the Swedish Research Council (VR) through the Contract 622-2008-1247 and the Swedish Government Strategic Research Area in Materials Science on Functional Materials at Linköping University (Faculty Grant SFO-Mat-LiU \# 2009-00971). LH and JJ acknowledge the Linköping Linnaeus Initiative LiLi-NFM, supported by the VR Grant 2008-6572. The authors are grateful for access to the Tandem Laboratory at Uppsala University. 


\section{References}

[1] J.D. Corbett, H.S. Marek, Inorg. Chem. 22 (1983) 3194.

[2] T.B. Massalski, J.L. Murray, L.H. Bennett, H. Baker, Binary Alloy Phase Diagrams, American Society for Metals, Metals Park, Ohio, USA (1986).

[3] J. Köbler, J.-M. Welter, J. Less-Common Met. 84 (1982) 225.

[4] International Centre for Diffraction Data, Tantalum Hydride $\left(\beta-\mathrm{TaH}_{2}\right), \mathrm{PDF}$ no: 03-0923.

[5] International Centre for Diffraction Data, Tantalum Hydride $\left(\mathrm{TaH}_{0.93}\right)$, PDF no: 39-1329.

[6] Y. Fukai, M. Mizutani, Mater. Trans. (Jap), 43 (2002) 1079.

[7] A.L. Allred, J. Inorg. Nucl. Mater., 17 (1961) 215.

[8] K. Sarakinos, J. Alami, S. Konstantinidis, Surf. Coat. Technol. 204 (2010) 1661.

[9] S. Hüfner, Photoelectron Spectroscopy: Principles and Applications, third ed. SpringerVerlag: Berlin, Germany, 2010.

[10] H.J. Whitlow, G. Possnert, C.S. Petersson, Nucl. Instrum. Methods Phys. Res. Sect. B: Beam Interact. Mater. At. 27 (1987) 448.

[11] J. Jensen, D. Martin, A. Surpi, T. Kubart, Nucl. Instrum. Methods Phys. Res. Sect. B: Beam Interact. Mater. At. 268 (2010) 1893.

[12] J.F. Moulder, W.F. Stickle, P.E. Sobol, K.D. Bomben, in: J. Chastain (Ed.), Handbook of X-ray Photoelectron Spectroscopy, Perkin-Elmer Corporation, Physical Electronics Division, Eden Prairie, MN, USA, 1992. 
[13] W. Kern, V.S. Ban, in: J.L. Vossen, W. Kern (Eds.), Thin Film Processes, Academic Press, Inc, New York, NY, USA, 1978, pp. 257-331.

[14] P.E. Pehrsson, F.G. Celii, J.E. Butler, in: R.F. Davis (Ed.), Diamond Film and Coatings, Noyes Publications, Park Ridge, NJ, USA, 1993, pp. 68-146.

[15] J. Alami, P. Eklund, J.M. Andersson, M. Lattemann, E. Wallin, J. Bohlmark, P. Persson, U. Helmersson, Thin Solid Films 515 (2007) 3434.

[16] A. Jiang, T.A. Tyson, L. Axe, L. Gladczuk, M. Sosnowski, P. Cote, Thin Solid Films 479 (2005) 166.

[17] P.N. Baker, Thin Solid Films, 14 (1972) 3.

[18] P.T. Moseley, C.J. Seabook, Acta Crystallogr. B 29206 (1973) 1170.

[19] H.J. Lee, J. Appl. Phys., 57 (1985) 4037.

[20] C. Paturaud, G. Farges, M.C. Sainte Catherine, J. Machet, Thin Solid Films, 347 (1999) 46.

[21] J. Alami, P.O.Å. Persson, D. Music, J.T. Gudmundsson, J. Bohlmark U. Helmersson, J. Vac. Sci. Technol. A 23 (2005) 278.

[22] M. Samuelsson, D. Lundin, J. Jensen, M.A. Raadu, J.T. Gudmundsson, U. Helmersson, Surf. Coat. Technol. 205 (2010) 591.

[23] N. Matsunami, Y. Yamamura, Y. Itikawa, N. Itoh, Y. Kazumata, S. Miyagawa, K. Morita, R. Shimizu, H. Tawara, At. Data Nucl. Data Tables, 31 (1984) 1. 
[24] C. Kittel, Introduction to Solid State Physics, seventh ed., John Wiley \& Sons Inc., New York, NY, USA (1996) 


\section{Figure captions}

Figure 1. High-resolution XPS spectra of (a) $\mathrm{Cr} 2 p$ from a $\mathrm{Cr}-\mathrm{H}$ film deposited by HiPIMS with $20 \% \mathrm{H}_{2}$ in the plasma (solid line) and a Cr reference film grown by HiPIMS (dashed line), and (b) Ta 4f from of a Ta-H film deposited by HiPIMS with $20 \% \mathrm{H}_{2}$ in the plasma (solid line) and Ta reference film grown by HiPIMS (dashed line).

Figure 2. X-ray $\theta / 2 \theta$ scans of: a Ta reference film grown by HiPIMS (lower pattern), a Ta film deposited by HiPIMS and with $10 \% \mathrm{H}_{2}$ in the plasma (middle pattern), and a Ta film deposited by HiPIMS and with $20 \% \mathrm{H}_{2}$ in the plasma (upper pattern). The inset shows the $2 \theta$ region around the 002 peak for the deposited films.

Figure 3. X-ray $\theta / 2 \theta$ scans of: a Ta reference film grown by dcMS (lower pattern) and a Ta films deposited by HiPIMS and with $20 \% \mathrm{H}_{2}$ in the plasma (upper pattern).

Figure 4. SEM images obtained from $\beta$-Ta films, (a) $600 \mathrm{~nm}$ thick reference film deposited by HiPIMS, (b) $620 \mathrm{~nm}$ thick film deposited by HiPIMS and with $20 \%$ $\mathrm{H}_{2}$ in the plasma, (c) $660 \mathrm{~nm}$ thick reference film deposited by dcMS, and (d) 650 nm thick film deposited by dcMS and with $20 \% \mathrm{H}_{2}$ in the plasma.

Figure 5. SEM images obtained from $\alpha$-Cr films, (a) $410 \mathrm{~nm}$ thick reference film deposited by HiPIMS, (b) $400 \mathrm{~nm}$ thick film deposited by HiPIMS and with $20 \%$ $\mathrm{H}_{2}$ in the plasma, (c) $420 \mathrm{~nm}$ thick reference film deposited by dcMS, and (d) 360 nm thick film deposited by dcMS and with $20 \% \mathrm{H}_{2}$ in the plasma. 
Fig. 1

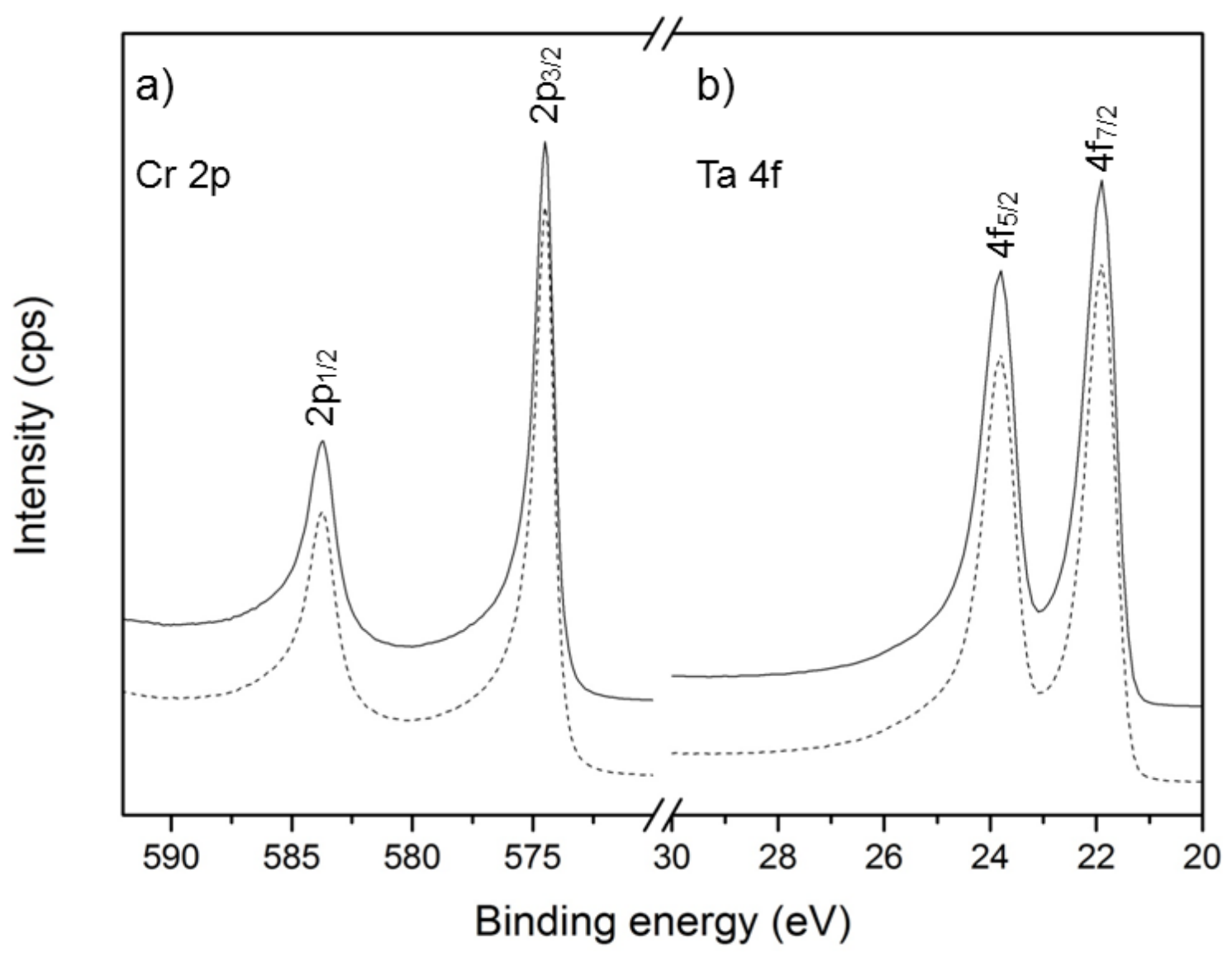


Fig. 2

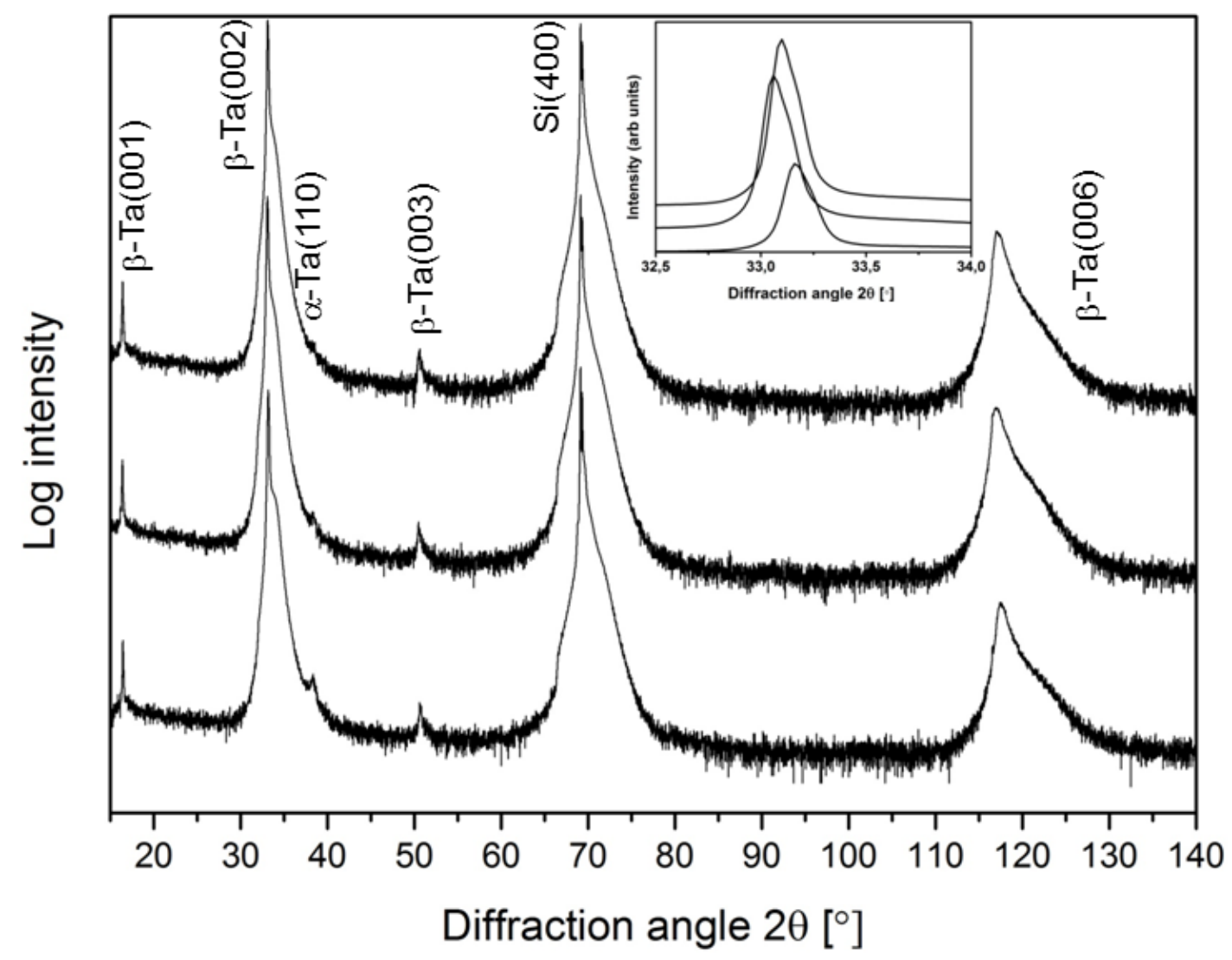


Fig. 3

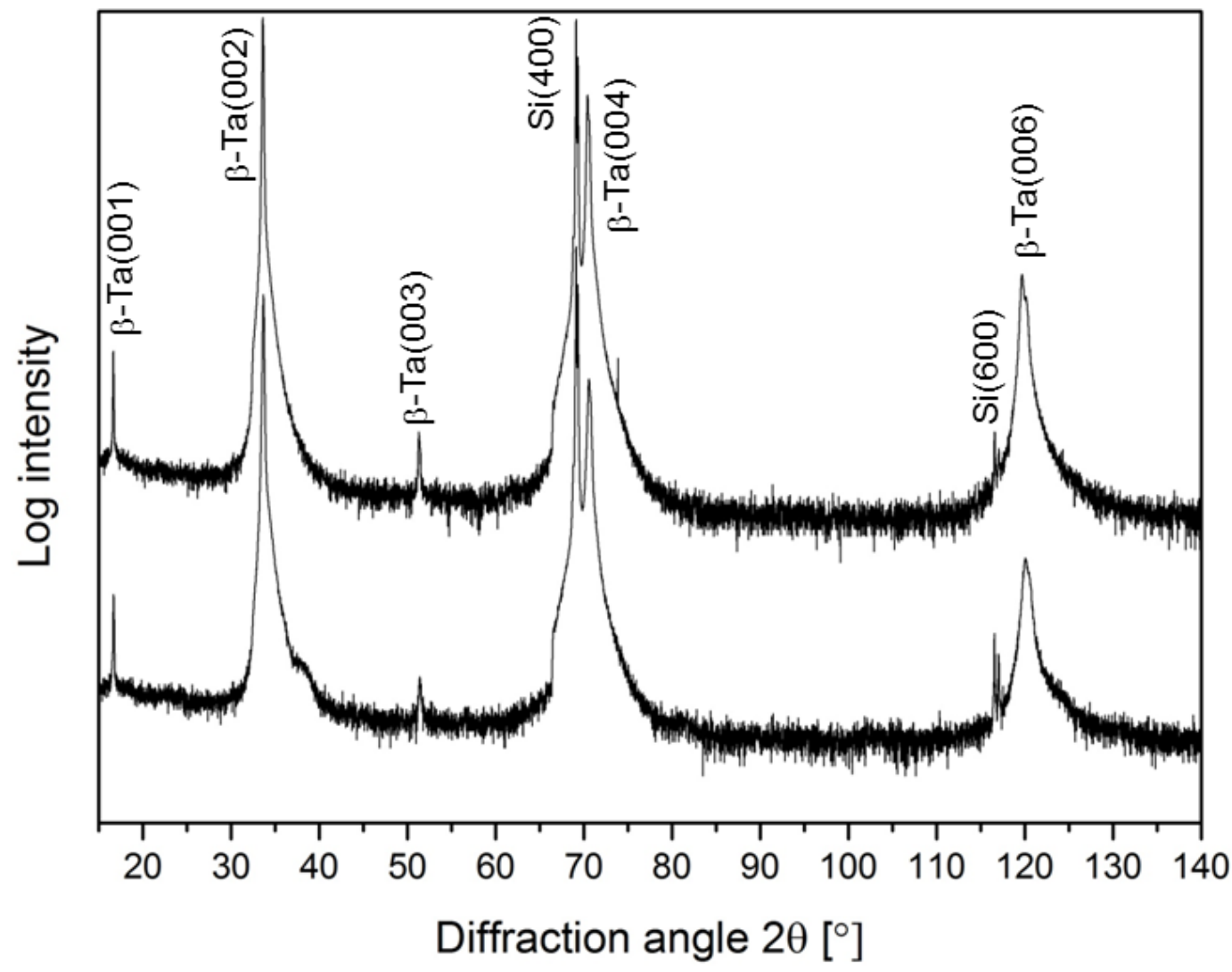


Fig. 4

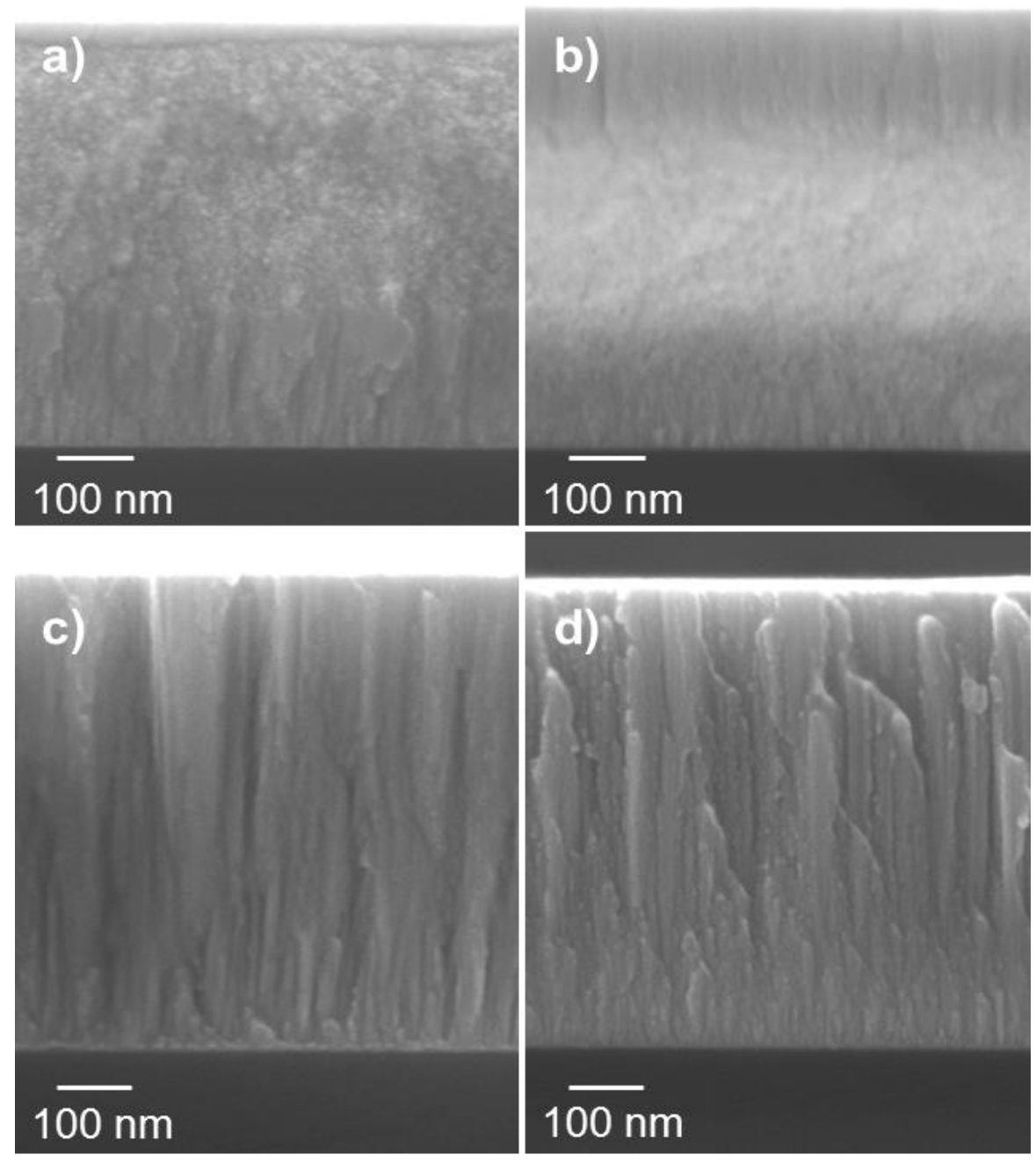


Fig. 5

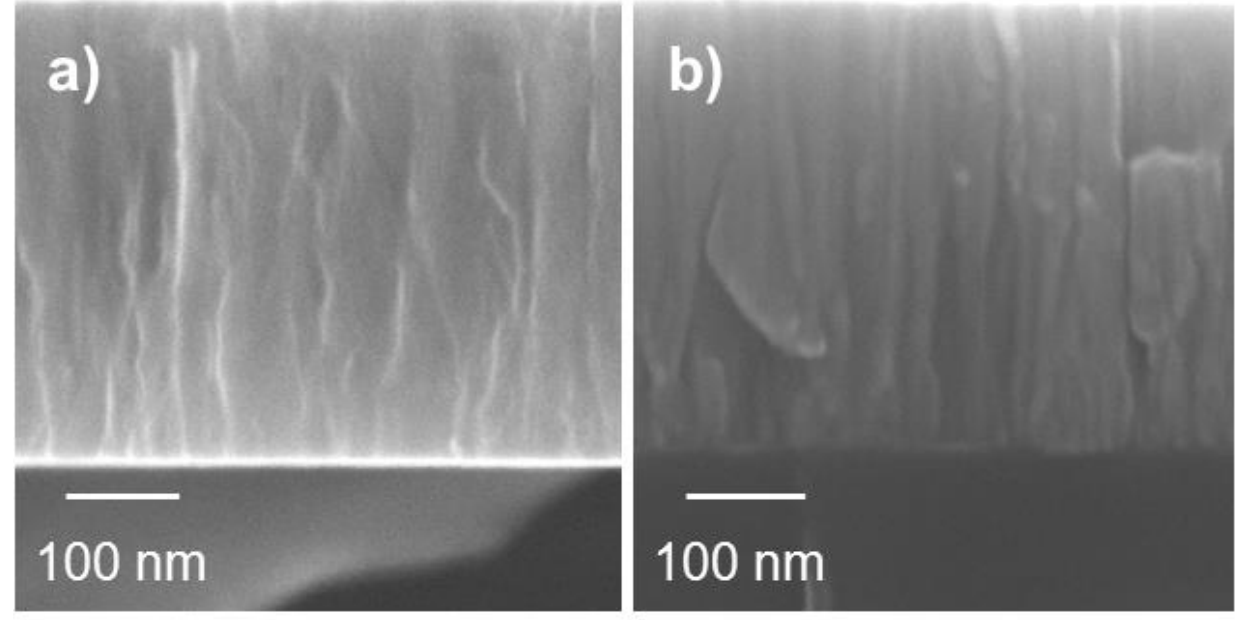

c)

d)

$100 \mathrm{~nm}$

$100 \mathrm{~nm}$ 